\title{
Alcances y límites de la política estatal en la economía social y solidaria.
}

\section{Análisis de dos emprendimientos productivos.}

Reaches and shortcomings of state policy towards the social and solidary economy. An analysis of two productive organizations

Yamila Crisci

FSOC/UBA- FADU/UBA

yamilacrisci@hotmail.com

Fernando Rada Schultze CONICET- FSOC/UBA- FLACSO

fernandorada@conicet.gov.ar

Fecha de recepción

24.7.13

Fecha de aceptación 6.3.14

\section{Resumen}

La última década no sólo registra un quiebre respecto al consenso que mantuviera un modelo social, económico y político excluyente durante los años 1990, sino que además representa una nueva manera de pensar las políticas sociales en la Argentina, esta vez sin la impronta residual del decenio neoliberal. Una de las esferas de la política estatal que se viera modificada es la que atañe al mundo laboral. Al mismo tiempo, el reciente decenio señalaría la recuperación de una serie de funciones clásicas del Estado perdidas años atrás, como pudiera ser su relación con la sociedad civil. Con una mirada crítica sobre las potencialidades y restricciones de las políticas sociales recientes, el siguiente artículo analiza el caso de dos emprendimientos de economía social y solidaria nacidos post crisis del 2001.

Palabras clave: Estado - políticas sociales - economía social y solidaria - cooperativismo - Argentina. 


\begin{abstract}
The last decade represents not just a breakdown against the consensus gained by a social, economical and political model during the 90's, but also a new way of thinking social policies in Argentina, now without the neoliberal's residual stamp of the previous decade. Labor issues experienced far reaching changes concerning public policy areas. At the same time, the last decade involves the recovery of many classical (Wellfare) State functions lost years before, such as its relationship with civil society.

Through a critical view regarding recent social policy shortcomings and potentialities, this article conducts two case studies of what is usually known as social and solidarity economy, born after the 2001 crisis.
\end{abstract}

Keywords: State - social policies - social economy cooperative enterprises - Argentina.

\title{
I. Introducción
}

Diversos autores acuerdan que en los últimos años la estructura social, económica y política de nuestro país experimentó cambios sin precedentes (por ejemplo Aronskind, 2001; Murmis y Feldman, 1993). Entre estas transformaciones se encuentran las que conocieron el trabajo, la economía y el Estado, temas que atañen al presente artículo. En este sentido debe señalarse que uno de los hechos que alteró las sociabilidades habría sido la pérdida masiva de empleos y la flexibilización laboral características de la década de 1990. Históricamente la sociedad argentina se había distinguido por su movilidad social ascendente resultante del acceso a dos instituciones: la educación y el trabajo. El trabajo fue durante gran parte del Siglo XX un forjador de identidades. La sociedad argentina se encontraba cohesionada al calor de la figura del trabajador asalariado formal, lo que significó, durante casi un siglo, ser sujeto de derechos y ciudadano. Ser trabajador/a representaba en nuestro país contar con aportes jubilatorios, con un sistema de salud, encontrarse respaldado por una organización sindical, tener vacaciones pagas, entre otros aspectos. Era, en síntesis, ser alguien, tener una identidad individual y social.

Con el advenimiento del modelo neoliberal en la región, esta matriz socioeconómica se perdería. Un cuarto de siglo de medidas de corte excluyente, agravado en la década de 1990, dejaría hondas marcas en la estructura productiva argentina y en la distribución del ingreso, corroyendo mecanismos de ascenso social y segmentando, maginando y pauperizando a amplios sectores poblacionales (Bankirer, 2010; Torrado, 2007). 
En ese mismo contexto, el Estado también fue otra de las instituciones que vio reducida su actividad y reestructuradas o perdidas sus facultades clásicas, entre las que debe ubicarse el ser asociado a una entidad con capacidad de otorgar o denegar identidad y ciudadanía. Al mismo tiempo, las reducidas acciones políticas que llevó a cabo han sido caracterizadas como focalizadas y reparatorias (Pautassi et al, 2008). No obstante, años más tarde, el Estado recuperaría algunas de sus facultades tradicionales; por ejemplo, los cambios en su dimensión, formas de intervención y relaciones con los diferentes actores. El Estado volvería a ser visto como núcleo al cual dirigir las demandas sociales en tanto entidad capaz de otorgar identidad y derechos; como institución con poder y autoridad de reconocer ciudadanía (Vilas, 2005).

A pesar de los cambios experimentados, la recuperación de instituciones como el trabajo y el Estado son recientes. En este mismo período, en el que estas entidades se caían y volvían a construirse, un abanico de modos alternativos de economía tomó fuerza, en conjunto englobados bajo la denominación de economía social y solidaria. Diferentes creaciones de puestos laborales como emprendimientos productivos, autoempleo, cooperativas y fábricas recuperadas por sus trabajadores y trabajadoras, fueron expresiones que los actores hallaron para hacer frente a la paupérrima situación que debían afrontar, al tiempo que reivindicaban y fortalecían su posición social e identidad como sujeto trabajador.

En esa línea de análisis, Caracciolo Basco y Foti Laxalde (2003) destacan la dimensión relacional y asociativa de las unidades que interactúan en la producción e intercambio de bienes y servicios, aspectos nodales dentro de la lógica social, económica y laboral en el marco de la incorporación de políticas públicas que encuentran en la economía social un modelo alternativo para reconstruir el tejido social. Concordantemente, y retomando sendas de trabajo propuestas anteriormente ${ }^{1}$, enfocamos dos casos de emprendimientos de economía social y solidaria y los analizamos de manera comparativa: el emprendimiento productivo "Sol de pan" y la cooperativa "Estilo Diversa LGBT". Prestaremos atención al desarrollo de estos emprendimientos laborales, el rol jugado por el Estado a través de políticas que promuevan estas actividades, y discutiremos sobre las perspectivas que experiencias como éstas ofrecen para generar trabajo genuino en tanto formador de identidades sociales.

\section{Metodología}

El presente artículo presenta un diseño cualitativo de estudio de caso. Siguiendo la lectura de Flyvbjerg (2011), el estudio de caso consiste en un análisis intensivo de una unidad individual (sea esta una comunidad o una persona), que permite obtener información confiable acerca de la clase más amplia a la que la unidad en cuestión pertenece: la comprensión de las causas de un fenómeno y su contexto. Asimismo, Flyvberg carac- 
teriza entre los puntos fuertes de este tipo de análisis su profundidad y su alta validez conceptual, como así también la capacidad que tiene esta metodología de brindar información útil en las etapas preliminares de una investigación, ya que proporciona hipótesis y abre interrogantes que podrán ser problematizados a la luz de un mayor número de casos (op.cit 301-314). Por lo tanto, aquí observaremos dos ejemplos que, a pesar de las diferencias que a priori presenta el mercado al que apuntan, propone casos relevantes de cooperativismo sobre los que echar luz y que, a posteriori, puede brindar herramientas para un estudio de mayor dimensión sobre las formas que adquiere la economía social y solidaria en la Argentina. Asimismo, ambos emprendimientos comparten la cualidad de componerse de actores marginados de la economía formal por diferentes motivos y, en algunos casos, sin haber participado nunca de un trabajo asalariado con las características que aquí le atribuimos. A su vez, se trata de ejemplos cooperativos que buscan interactuar con el Estado, al que demandan acciones precisas para mejorar la situación de su emprendimiento.

Con técnicas cualitativas como la observación no participante y las entrevistas en profundidad, nuestro trabajo estuvo guiado por el análisis del involucramiento estatal respecto de esos emprendimientos, incluyendo la existencia y alcance de acciones de promoción, así como un reconocimiento de la reconfiguración del rol del trabajo y de la figura del trabajador inserto en los emprendimientos productivos, marcando similitudes y divergencias entre ambas cooperativas.

\section{Lineamientos generales de la economía social}

La economía social toma significados diferentes según sea abordada desde los países centrales o periféricos (Mutuberría Lazarini, 2010). En nuestro caso, daremos importancia a la definición planteada desde los países latinoamericanos, denominados por la autora como periféricos. Para comenzar, es importante observar al individuo que integra las experiencias de este tipo de economías; generalmente se trata de individuos insertos en comunidades, familias y otros colectivos sociales que actúan no sólo en pos de la utilidad material sino también con vistas a la solidaridad y cooperación (Coraggio, 2007). De este modo, se toma a este tipo de economía como alternativa al sistema capitalista, en el que el interés principal reside en la obtención de ganancias sin una redistribución equitativa de la misma. Si bien la noción de mercado no desaparece, la concepción del mismo se modifica pasando a relaciones entre sus miembros que pretenden lograr integración y una distribución más igualitaria de los recursos y los beneficios.

Muchas experiencias en el campo de la economía social, surgieron como respuesta a las fallas en el sistema capitalista. Las sucesivas crisis del sistema generaron desempleo, exclusión social, empobrecimiento, marginación, entre otras consecuencias que dieron lugar a la asociación entre individuos y experiencias comunitarias conjuntas para so- 
brellevar y revertir esta situación y mejorar, de este modo, la calidad de vida. (Mutuberría Lazarini, 2010).

Resulta importante observar la reconfiguración del concepto de trabajo en las experiencias de economía social. En contraposición al rol del trabajador en el sistema capitalista de producción, en el que aquel, en tanto dueño de la fuerza de producción, es explotado por el capitalista que la compra en el mercado para producir plusvalor a partir de ella y quedarse con gran parte de las ganancias obtenidas, en las prácticas de economía social, el trabajador juega un papel preponderante con ejercicio de prácticas sociales que lo llevan a reapropiarse del control de su trabajo estableciendo modos solidarios de producción (Mutuberría Lazarini, 2010). De este modo, el dueño de la fuerza de trabajo y el de los medios de producción convergen en la misma figura que es la del trabajador (Vázquez Blanco y Rofman, 2011).

Cabe señalar que esta reconfiguración del sujeto y de la propia concepción del trabajador no se da al margen de la urdimbre social de una comunidad, donde el Estado ocupa un lugar fundamental. El papel desarrollado por el Estado en la economía social es un tema sobre el que debe interrogarse. Siguiendo a Coraggio (2005), podemos destacar que existe una corriente dentro de la economía social que aboga por la intervención del Estado para fomentar las prácticas de la economía social desde la administración pública y el sistema político. El Estado es quien puede, mediante el planeamiento de sus políticas, introducir formas de economía social como regulación de los mercados capitalistas. En este sentido, Vázquez Blanco y Rofman (2011) nos invitan a reflexionar -a partir del contexto económico, político y social que atraviesa actualmente nuestro país bajo un modelo de redistribución e inclusión social- sobre la economía social como componente fundamental del modelo económico presente. De este modo, y a diferencia del período de crisis desatado en 2001, donde los actores debieron utilizar diferentes estrategias para paliar los efectos de la misma, podremos pensar formas organizativas impulsadas desde el propio Estado y no desde los propios individuos.

\section{La economía social y el impulso del Estado}

La economía social se presenta como un modelo que procura reconstruir la integración desde lo colectivo por medio de un entramado solidario que potencie las redes sociales de los actores, puntualmente las territoriales. Esto en nuestro país se manifiesta en el modo en que las políticas son llevadas adelante. En la actualidad, las acciones del Estado no sólo buscan alejarse del modelo asistencialista característico de la década de 1990 -que sólo reproduciría intergeneracionalmente la pobreza- sino que llevan la huella del regreso del Estado ${ }^{2}$ allí donde en el decenio pasado había delegado sus funciones en las organizaciones del tercer sector. 
Es importante señalar que si bien las políticas inclusivas emanadas de este modelo solidario enfrentan profundas dificultades derivadas de la hegemonía de las relaciones de producción y de poder tradicionalmente ancladas en el modelo capitalista, y la constitución de subjetividades que de las mismas emana, no debe omitirse el giro que marcan con respecto al rol asumido por el Estado en su carácter de constitución del colectivo. Luego de una década de neoliberalismo donde los sujetos eran responsabilizados por su "incapacidad" para insertarse en el mercado y cuyas identidades se construían bajo el estigma de la desposesión, los años más recientes han estado marcados por la impronta de un Estado que problematiza dichas condiciones a la vez que acciona sobre las mismas. Una de las principales particularidades de los lineamientos de este modelo radica en el reconocimiento y la promoción de la potencia de las experiencias engendradas en contextos desiguales por medio de políticas que no se acotan a la reproducción de los sujetos desde y en los márgenes sino como protagonistas de la constitución de un colectivo.

La particularidad de estas experiencias radica en el carácter solidario en que dichas acciones se encuadran, ya que allí anida el reconocimiento de sus posibilidades. Donde con anterioridad eran las organizaciones no gubernamentales el intermediario para vehiculizar estrategias de supervivencia desde una lógica individualizada, hoy las diversas aristas de los programas (mediante apoyo a pequeños productores y talleres familiares, articulación de encadenamientos productivos, servicios de apoyo a la producción y asistencia a empresas autogestionadas) cristalizan la voluntad de un Estado que pretende retornar como principal articulador de lo social por medio de una lógica colectiva que promueve la integración por la vía de herramientas que procuran superar la asistencia. A diferencia de las miradas que se detienen en la crítica de los procesos de producción y trabajo de los sectores más pobres a partir de la ineficiencia de los mismos, las políticas actuales dan lugar al reconocimiento de los factores que los constituyen y estructuran pero no para descartarlos como posibilidad sino para intervenir desde la promoción de su productividad. De aquí se desprende una mirada que no escinde lo social y lo económico ya que imprime el objetivo de promover las experiencias de economía social y solidaria, no ahora en cuanto estrategias desde los márgenes sino que se integran como núcleos productivos.

No planteamos que ese tipo de políticas se lleven a cabo sin dificultades ya que las dimensiones vinculadas tanto a lo territorial como a los actores involucrados condicionan los grados de viabilidad y éxito de la economía social como modelo alternativo, pero destacamos que la singularidad del proceso actual radica el reconocimiento de los factores constitutivos de dichos procesos y el modo en que se interviene sobre los mismos, como el apoyo, la asistencia y la articulación.

El principio rector de estos emprendimientos se basa en la satisfacción de las necesidades y en la reproducción ampliada de la vida por medio del reconocimiento de las redes 
y capitales que constituyen su entramado, y no en la mera reproducción de la fuerza de trabajo. Así como el objetivo no se reduce a la ganancia, también se aparta de la figura del trabajador escindido de los medios de producción ya que ambos planos convergen y posibilitan, mediante el cooperativismo, la ampliación de grados de autonomía de los sujetos protagonistas del proceso de producción y comercialización.

Observamos aquí una política pública que procura promover la integración social ${ }^{3}$, no ya desde lo focalizado y en términos de transferencias de ingreso, sino que es transversal a otros mundos como el productivo y el del trabajo, entre otros, a partir del cual se da la posibilidad de reconstituir el entramado social.

Entrado en crisis el mundo tradicional del trabajo y dejando a la deriva a los sujetos, tanto en términos de satisfacción de necesidades básicas como de vías de construcción de identidad, las relaciones que se constituyen en el seno de la economía social trabajan sobre la inclusión pero desde una arista centrada en los valores comunitarios y asociativos en oposición a la lógica hiperindividualista de la economía mercantil.

Consideramos que la economía social y solidaria ofrece las posibilidades de articular desde una misma base políticas que vinculen la dimensión económica-productiva, la laboral y la social, sentando las condiciones para la promoción de un sentido de acción y pertenencia colectiva. Esto se hace posible de la mano de un Estado que no llega a la sociedad desde acciones fragmentadas y fragmentarias sino con un sentido de amplio alcance. Es por medio de las mismas que el trabajo como vía de inclusión social parece poder reconstituirse con base en un entramado de relaciones que se resignifica en su proceso de constitución.

\section{El emprendimiento productivo "Sol de Pan"}

El primero de los casos analizados es el de "Sol de Pan", una ecopanadería que elabora sus productos de forma artesanal y trabaja con un horno que sólo utiliza energía solar. Ubicada en el barrio "Un techo para todos", aledaño al barrio 22 de Enero en Ciudad Evita, Partido de La Matanza, Gran Buenos Aires, la panadería representa un ejemplo de las experiencias de estas modalidades de economía social y solidaria a las cuales referimos en la sección anterior.

El emprendimiento nació en el año 2006, a partir de la donación de un horno solar llamado "Inti Raymi"” otorgado por la Fundación "Hábitat para la Humanidad", que se encuentra realizando diversas actividades en el barrio desde hace algunos años. La particularidad de "Inty Raymi" es que posee paneles solares que reflejan en el horno de tamaño industrial con tapa de vidrio y puede alcanzar una temperatura de hasta 180 grados, permitiendo el horneado de productos sin gasto de energía más que el abastecimiento solar. 
Una segunda fundación, Concordia, realizó en el barrio una capacitación sobre micro energía, que permitió dar inicio al emprendimiento. Esta misma fundación se hizo cargo de la materia prima para lograr la primera producción con el fin de llevar un "pan dulce" a cada familia del barrio en Navidad. Es decir que el emprendimiento en cuestión, comienza como una economía doméstica de autoabastecimiento para el período navideño. A partir de esa primera producción varias fundaciones y personas que trabajaban en el barrio comenzaron a encargar pedidos más grandes y la experiencia productiva fue creciendo gradualmente.

El equipo de "Sol de Pan" se compone de entre 3 y 5 trabajadores. Un panadero aporta sus conocimientos técnicos y el resto se encarga de las compras de materia prima, elaboración, promoción, venta, y entrega de los productos. Una de sus integrantes relató, en detalle, el rol de cada uno en la jornada laboral:

"Uno se encarga de ir a comprar y Blanca y yo nos encargamos de elaborar. Preparamos todo un día antes, la pasta $y$ al otro día la masa. En la época de pan dulce trabajamos el día completo para poder terminar con todo. Viene por la mañana Humberto, mi socio (porque nosotros nos decimos así, socios), que abre el horno, lo limpia, limpia los paneles y prepara todo. Nosotras nos ponemos a elaborar y él va controlando al horno. Eso lleva un tiempo largo, pero ya nos manejamos de esa forma. El envasado también lo hacemos nosotros, todo empieza y termina acá. Todo funciona acá, en mi propia casa”. (Entrevista realizada a Rita, miembro de la eco-panadería "Sol de Pan”, Octubre de 2011)

Este fragmento de la entrevista a Rita arroja varias reflexiones en relación a lo que venimos desarrollando. En primer lugar, observamos la marcada división de tareas entre los miembros del grupo de trabajo para llevar a cabo la jornada laboral. En segundo lugar, el manejo de los horarios de trabajo según la época del año y las exigencias de entrega de los productos. Y, por último, la referencia de la entrevistada a la realización completamente artesanal de los productos de la panadería. Estas observaciones dan cuenta, precisamente, del rol protagónico del trabajador en el proceso productivo y, además, de su participación en el mismo. En este caso particular, y teniendo en cuenta que la panadería es llevada adelante por pocas personas, los trabajadores participan del proceso completo tanto de producción como de venta de sus productos sin tener que rendir cuentas de sus tareas a patrones, encargados o jefes. Ellos son -al mismo tiempo- quienes dan y ejecutan las órdenes para que el emprendimiento siga adelante, re apropiándose constantemente del control de su trabajo. 
Con el correr del tiempo, fueron agregando variedades de productos además de "pan dulce" y comenzó la elaboración de pan casero, chipá, rosca de pascua, sopa paraguaya, entre otros. Debido a sus características, el horno es utilizable solo en periodos del año en los que hay sol aprovechable como energía -desde octubre/noviembre hasta el mes de marzo. Ante la dificultad que significaba continuar con la producción en los meses restantes, en el tercer año de existencia de "Sol de Pan", la Asociación Civil "Cirujas" les ofreció un microcrédito otorgado por el Ministerio de Desarrollo Social en el marco del Programa Banco Popular de la Buena Fe que es una modalidad del componente "Fondos Solidarios" del Plan Nacional "Manos a la obra", dentro del programa "Argentina Trabaja". A través del otorgamiento de este microcrédito, el emprendimiento pudo obtener un horno panadero que se alimenta con gas en garrafa, para utilizar los días de invierno o de lluvia en los que "Inti Raymi” no es aprovechable. De este modo, el emprendimiento puede funcionar y seguir comercializando sus productos durante todo el año.

Éste es el primer momento en el que detectamos la presencia estatal en relación al emprendimiento. Se advierte una participación estatal indirecta, mediada por otros actores (fundaciones) que facilitaron el acceso al microcrédito y limitada únicamente a este hecho, sin extenderse más allá de esta intervención puntual de prestación monetaria. Ello no obstante es posible inferir una clara intención del Estado en favorecer emprendimientos de economía social y solidaria a través de los programas llevados a cabo por el Ministerio de Desarrollo Social. Incluso, a través del Instituto Nacional de Asociativismo y Economía Social (INAES) -organismo que ejerce las funciones que le competen al Estado en materia de promoción, desarrollo y control de la acción cooperativa y mutual- se favorecen emprendimientos de este tipo. Podemos concluir, entonces, en una limitada participación estatal respecto a "Sol de Pan".

Cabe señalar que la panadería no resulta, aún, el principal ingreso de sus trabajadores sino que lo toman como un trabajo adicional y el dinero que va ingresando lo reinvierten para que la panadería siga creciendo y -en caso de que haya un mayor excedente- lo dividen en partes iguales.

\section{El caso de la cooperativa textil "Estilo Diversa LGTB"}

Ubicada en el barrio de Balvanera de la Ciudad Autónoma de Buenos Aires, próxima a Plaza Miserere, se encuentra la cooperativa textil Estilo Diversa, perteneciente a la Federación Argentina de Lesbianas, Gays, Transexuales y Bisexuales (FALGTB), creada a fines de 2010 como un emprendimiento desde una organización de la sociedad civil que buscaba financiamiento estatal. Surgida como una inquietud de la FALGTB, la incipiente cooperativa tuvo como objetivo incorporar -aunque no de manera exclusiva- a personas transexuales y travestis, quienes por su condición sexual veían cercenadas las posibilidades de obtener otro tipo de trabajo, siendo con frecuencia la prostitución el 
único medio de subsistencia. De todas maneras sus trabajadores/as actuales son diversa orientación sexual.

Más allá de que la idea surgiera desde un movimiento social, el rol del Estado fue clave en la gestación de la cooperativa. Entre septiembre y octubre de 2010 miembros de la FALGTB se reunieron con personal del INAES, del Ministerio de Desarrollo Social y del Ministerio de Trabajo. De estas entrevistas se logró un primer financiamiento anual. El subsidio estipulaba el otorgamiento de $600 \$$ mensuales a lo largo de un año para cada trabajador/a. Parte del convenio además garantizaba un mínimo de quince personas a las que se les brindaría un puesto de trabajo. Luego de ese tiempo se concedió un subsidio no reintegrable para la adquisición de maquinaria, de $\$ 60.000$, previéndose que la ampliación de la producción llevaría a un incremento de los puestos de trabajo. La experiencia debió enfrentar una variedad de escollos y desafios, especialmente referidos al empleo y la prestación laboral, así como a la del colectivo transgénero.

Por ejemplo, muchas de las personas que trabajan en la cooperativa, específicamente las travestis, rara vez comienzan su jornada laboral en la mañana. Lejos de una falta de compromiso o interés, la respuesta frecuente se encuentra en la necesidad económica de continuar ejerciendo la prostitución ante el bajo monto de remuneración que perciben del trabajo en la cooperativa, considerablemente inferior al ingreso que obtenían del trabajo en la calle. Al mismo tiempo el ejercicio de este tipo de trabajo, obviamente informal, se caracteriza por la carencia de redes sociales formales de apoyo o cualquier expresión de seguridad social; en consecuencia las trabajadoras sexuales se ven expuestas a mayores condiciones de violencia y marginalidad, producto de la discriminación derivada de la homofobia.

Además de la frecuente violencia y la discriminación, una persona transgénero, cuando descubre su identidad, debe encarar la adecuación de su cuerpo a su sentir. Es éste un proceso continuo, que requiere de operaciones varias y la ingesta de hormonas. La persona debe ingerir hormonas cotidianamente y de manera periódica cambiar las prótesis o siliconas. Esto tiene no sólo un costo económico, sino que el operarse, sobre todo si se trata de una intervención clandestina, demanda un tiempo de reposo, lo cual se traduce en días sin trabajar y por ende en días sin un centavo. La prostitución entonces sigue presentándose como un modo alternativo -y económicamente seductor- de trabajo.

Otra dimensión donde podemos encontrar tensiones es respecto al mercado y la competitividad. Una preocupación constante que ciñe al quehacer de la cooperativa es la de cómo lograr hacer frente a la competencia de otras empresas que cuentan con diferente capacidad productiva. En el caso de estudio el modo que encontraron es el de obtener un mercado cautivo. A pesar de que la cooperativa inicialmente quiso apuntar a una oferta exclusivamente textil, la intención de sus integrantes es expandir la variedad de los productos. De esta manera se busca vender objetos específicos para así competir en 
una economía de mayor dimensión. Entre estos productos se ubica la producción de accesorios relacionados con temáticas de la diversidad sexual. En la actualidad elementos como ser tazas, llaveros, pulseras, banderas, y otras cuestiones vinculadas al orgullo LGTB, rara vez son producidos por personas pertenecientes al colectivo homosexual. Por lo tanto se persigue una producción de la diversidad y para la diversidad.

Lo acotado y reciente de esta cooperativa conduce a los y las militantes de la Federación a buscar otras actividades redituables para el sector vulnerado que representan. Entre ellas podemos nombrar el intento de la creación de una oficina de empleo amigable a la diversidad. Su objetivo es sensibilizar a las empresas sobre las problemáticas de las personas transexuales por la marginación y la discriminación a la que están expuestas (no finalización de sus estudios, abandono del hogar en la adolescencia, problemas de salud ante operaciones clandestinas, entre otras) y el modo en el que las ayudarían en caso de contratarlas. Este trabajo consistiría en capacitaciones al personal de diferentes empresas llevado adelante por los propios activistas. Además se buscaría el modo de seducir la inclusión de personas transgénero y transexuales por medio de artilugios como ser la desgravación impositiva. Estas charlas y capacitaciones a cargo de personas de la diversidad serían financiadas por el Ministerio de Trabajo.

Se desarrollaron también cursos de capacitación de cuidadores de adultos/as mayores -actividad que ya ha entregado diplomas a sus primeras egresadas. Estos cursos sin embargo presentan limitaciones, entre ellas el bajo monto de la remuneración percibida por las cuidadoras tras el aprendizaje, y la extensión de la semana laboral. Por otro lado debe considerarse que las travestis provienen principalmente del noroeste argentino pero la capacitación se realiza únicamente en la Ciudad de Buenos Aires, con lo cual hasta podría llegar a estimularse el éxodo regional al tiempo que no se erradicaría el machismo y la homofobia de sociedades sumamente patriarcales. ${ }^{5}$ Además debiera haber sido considerado que las personas mayores, que serán cuidadas por una joven transgénero, han sido socializadas en contextos que estigmatizaban la homosexualidad considerándola una enfermedad o perversión. Por lo tanto puede existir un rechazo de la misma persona adulta hacia su cuidadora.

Por último, debe señalarse que las travestis que egresan de estos cursos no cobran un sueldo, sino que su remuneración es considerada un subsidio/beca, por lo que no existe esa relación capaz de otorgar identidades y derechos, como es la brindada por un trabajo asalariado formal.

\section{Reflexiones finales}

Corresponde en esta sección indagar crítica y comparativamente las características, desarrollos, similitudes y desemejanzas en los dos casos considerados, como así también la 
participación del Estado en ellos y el alcance de las políticas llevadas a cabo en materia de trabajo y cooperativismo.

Una manera de aproximarnos a las metas planteadas al inicio de este escrito es a través de la idea del trabajo en tanto esfera identitaria; como uno de los tantos " ámbitos de socialización significativos, que constituyen espacios de construcción de formas identitarias" (Kossoy 2003: 67). Tomando esta premisa a lo largo de estas líneas problematizamos en torno a los cambios experimentados en años recientes en lo relativo al trabajo, la economía y el Estado. En este sentido, tras la pérdida masiva de empleos y la flexibilización laboral en décadas pasadas, se dejó de lado esta concepción del trabajo imperante a lo largo del siglo XX. Las medidas económicas neoliberales presentes en la historia nacional reciente corroyeron mecanismos de ascenso social segmentando, marginando y pauperizando a amplios sectores poblacionales, lo cual empujaría a los actores a valerse por sí mismos; motivación que, entre otros resultados, daría lugar al nacimiento de diferentes emprendimientos económicos alternativos. Años más tarde el Estado recuperaría algunas de sus capacidades tradicionales -por ejemplo cambios en su dimensión, formas de intervención y relaciones con los diferentes actores, ente los que se incluye la sociedad civil, a la que pertenecen las organizaciones de economía social y solidaria.

A partir de estos elementos de encuadramiento, el estudio de dos emprendimientos económicos nos permitió observar sus principales actividades y la presencia estatal en ellas, registrándose algunas divergencias entre ambos casos. Ante todo, respecto al surgimiento de ambas experiencias, el emprendimiento productivo "Sol de Pan" surge como un proyecto de economía doméstica para un período específico del año y luego crece con el tiempo para pasar a ser un proyecto permanente. En cambio, "Estilo Diversa" es pensado desde una organización de la sociedad civil para incluir a personas que por su condición sexual (transexuales, travestis) veían dificultadas las posibilidades de ejecutar algún tipo de trabajo por fuera de la prostitución. Con relación a la intervención estatal, en "Sol de Pan" el Estado se hizo presente una vez comenzado el emprendimiento y ante un caso puntual (a través de un microcrédito) sin sostener su participación a lo largo del tiempo. En "Estilo Diversa" el Estado intervino desde un primer momento, siendo su principal fuente de financiamiento. En cuanto a las ganancias arrojadas, observamos un punto de contacto en ambos emprendimientos. Por un lado, para los integrantes de "Sol de Pan" los ingresos no resultan suficientes como para convertirse en el principal sostén de las familias nucleadas alrededor del emprendimiento; en "Estilo Diversa" los ingresos también resultan acotados y sus integrantes terminan recurriendo a su antiguo modo de subsistencia. Adicionalmente, debe señalarse en ambos casos la falta de prestaciones sociales: aportes jubilatorios, obra social, entre otras.

A pesar de tratarse de modos alternativos de organización económica, no debe dejarse de lado el hecho de que ambos emprendimientos se inscriben en una sociedad 
de mercado. Aunque en ellas sus integrantes no piensan su desempeño en términos de costo-beneficio ni ganancia, lo cierto es que estas cooperativas, al ser parte de un sistema capitalista, deben regirse por ciertas pautas de mercado, apuntando, por ejemplo, a la rentabilidad de sus productos. En el caso del emprendimiento "Sol de Pan", vemos cómo su labor resulta más próxima a una economía de subsistencia e informal. Sin grandes planteamientos estratégicos, buscan que la publicidad de su producción sea transmitida de persona a persona por medio de los clientes-vecinos. Recientemente, apremiados por la necesidad de expandir el negocio, sus integrantes realizaron cursos para promocionar sus productos en internet. Diferente es el caso de la cooperativa "Estilo Diversa" que desde un primer momento buscó diversificar la producción al tiempo de apuntar -y generarse- un mercado cautivo: la población lesbiana, gay y transexual.

Con esas limitaciones, debe reconocerse que ambos emprendimientos han generado vínculos asociativos entre sus integrantes y una resignificación del trabajo tras el impacto de la década neoliberal. Sin embargo, estos efectos podrían modificarse en el corto plazo, ya que es discutible que las acciones del Estado sean suficientes para la reproducción de la propia vida de los actores implicados, como así también de la reproducción de la fuerza de trabajo.

Por último cabe señalar que mientras la normalización de la informalidad del trabajo en algunos sectores conllevó a la naturalización de relaciones de producción y trabajo precarias, observamos aquí el carácter fundante de un modelo cuyo impacto sobre la constitución de los sujetos será un elemento fundamental ya que descansa sobre el principio de contemplar dichas formas de producción no desde aquello de lo que carecen sino de las potencialidades que engendran. Al ser el campo de la economía social un terreno en constante organización y crecimiento, la introducción de estas nuevas formas debería efectuarse de manera gradual, de modo de permitir la observación de sus efectos y extenderlas. Será trabajo futuro recuperar las observaciones aquí planteadas para indagar en los límites y alcances de otros emprendimientos del amplio campo de la economía social, para lo cual este artículo pretendió ser un aporte. 
${ }^{1}$ El presente trabajo surge como corolario del seminario Economía Política dictado por Alejandro Rofman para la Maestría en Políticas Sociales de la Universidad de Buenos Aires.

${ }^{2}$ Una de las líneas de acción que evidencia dichos principios es el Plan Nacional Desarrollo Local y de Economía Social "Manos a la obra" por medio del cual es el Estado quien, a través del otorgamiento de financiamiento y asistencia técnica, reconoce la potencialidad de los proyectos generados.

${ }^{3}$ Siguiendo a Estela Grassi (2007) encontramos la complejidad de nociones como "inclusión" e "integración" presentes en las políticas estatales. Como señala la autora, la integración y la adaptación fueron hasta hace algunos años nociones valoradas negativamente por tratarse de sinónimos de adecuación a las normas imperantes (Grassi, 2007: 7). Pese a ello, entendemos al concepto de integración social en términos de inclusión de un determinado sector -anteriormente excluido- al tejido social, puntualmente a través de las experiencias de economía social y solidaria mencionadas.

${ }^{4}$ El horno proviene de una donación del Rotary Club de Inglaterra, y en principio estaba destinado a otro lugar, pero debido a demoras para su ingreso al país, los dueños del otro microemprendimiento no lo necesitaron y así fue como llegó a manos de los habitantes del barrio 22 de Enero.

${ }^{5}$ Puede recordarse por ejemplo la gobernación de Salta fue una de las pocas jurisdicciones que se resistió a aplicar el nuevo programa de educación sexual. 


\section{Referencias Bibliográficas}

Aronskind, Ricardo (2001): ¿Más cerca o más lejos del desarrollo? Transformaciones económicas en los '90, Buenos Aires: Libros del Rojas, $N^{\circ} 2$.

Bankirer, Mónica (2010): “Composición de la población y envejecimiento: del 'país de inmigrantes' al "país de adultos mayores"' en Torrado, S: El costo social del ajuste. Argentina 1976-2002. Buenos Aires, Edhasa Tomo I: 187-206.

Caracciolo Basco, Mercedes y Foti Laxalde, María del Pilar (2003): Economía Solidaria y Capital Social. Buenos Aires, Editorial Paidos.

Castel, Robert (1997): La metamorfosis de la cuestión social. Una crónica del asalariado, Buenos Aires, Paidós.

Coraggio, José Luis (2005): “La Economía Social como vía para otro desarrollo social.” Extraído de http://www.redetis.iipe.unesco.org/publicaciones/economiasocialcoraggio.pdf Consultado: 02.12.2012 [último acceso: 17.03.2014].

Flyvbjerg, Bent (2011): “Case study”, en Norman K. Denzin and Yvonna S. Lincoln, eds., The Sage Handbook of Qualitative Research, Thousand Oaks, CA: Sage, 2011, Cap. 17: 301-316.

Grassi, Estela (2007): "La política social, las necesidades sociales y el principio de la igualdad: reflexiones para un debate 'postneoliberal", , en Congreso Latinoamericano y Caribeño de Ciencias Sociales, FLACSO-SENPLADES, Quito, Octubre 2007.

Kossoy, Alicia (2003): “Triangulación de técnicas de relevamiento: alcances y limitaciones”, en Lago Martínez, S; Gómez Rojas, G y Mauro, M (cords.) En torno de las metodologías: abordajes cualitativos y cuantitativos, Buenos Aires, Proa XXI: 63-72.

Merlinsky, Gabriela y Rofman, Adriana (2005): “Los programas de promoción de la Economía Social: ¿una nueva agenda para las políticas sociales?” En Forni, F: Caminos solidarios de la economía argentina. Buenos Aires. Ediciones Ciccus: 161-190.

Murmis, Miguel y Feldman, Silvio (1993): "La heterogeneidad social de la pobreza”, en Minujin, A (comp.): Cuesta abajo, Buenos Aires, UNICEF-Losada: 45-92.

Mutuberria Lazarini, Valeria (2010): "El campo de la Economía Social en debate" en Garcia, A. (coord.): Repensando la Economía Social. Buenos Aires. Ediciones del CCC. Centro Cultural de la Cooperación Floreal Gorini: 11-28. 
Oszlak, Oscar y O’ Donnell, Guillermo (1976): Estado y políticas estatales en América Latina: hacia una estrategia de investigación, Documento Cedes / G. E. Clacso No 4, Buenos Aires.

Pautassi, Laura; Arcidiácono, Pilar; Royo, Laura; Straschnoy, Mora y Carmona Barrenechea, Verónica (2008): “Tensiones en un marco de crecimiento económico. La política social pendiente”, en Centro de Estudios Legales y Sociales (CELS) VV.AA: Derechos Humanos en Argentina. Informe 2008. Buenos Aires, Siglo XXI: 283-306.

Soldano, Daniela y Andrenacci, Luciano (2005): “Aproximación a las teorías de la política social a partir del caso argentino”, en Andrenacci, L. (comp.): Problemas de política social en la Argentina contemporánea. Buenos Aires: Prometeo/UNGS: 51-79.

Torrado, Susana (2007): “Estrategias de desarrollo, estructura social y movilidad”. En Torrado, S (comp.): Población y bienestar en la Argentina del primero al segundo bicentenario. Buenos Aires, Edhasa, Tomo I: 31-68.

Vázquez Blanco, Juan Manuel y Rofman, Alejandro (2011): “Al cierre del Bicentenario dos modelos de país en disputa” en Fraschina, J. y Vazquez Blanco, J. M. (comp.) Aportes de la Economía Política en el Bicentenario. Prometeo Libros: 23-50

Vilas, Carlos M. (2005): “¿Estado víctima o promotor? El debate sobre soberanía y autonomía en el capitalismo Globalizado" en Vilas, C; Forcinito, K. et al: Estado y política en la Argentina Actual. Buenos Aires: Prometeo. 